\title{
Le « bien vieillir 》 : concepts et modèles
}

> Depuis quelques années, l'image associée au Marcellin Gangbè, Francine Ducharme phénomène du vieillissement est plus positive: on parle de «bien vieillir», de «vieillissement réussi » ou de «vieillir en santé ». Aucun consensus ne se dégage encore sur ce concept provocateur et stimulant. Dans cette synthèse des principaux écrits, nous présentons un point de vue sur les acceptions et modèles du «bien vieillir». Ainsi, il apparaît que le contenu du concept varie en fonction du contexte culturel, de la perspective des acteurs et selon les approches. Plusieurs modèles sont aussi identifiés: les uns, unidimensionnels, envisagent le bien vieillir sous l'angle d'un domaine scientifique particulier; les autres, multicritères, adoptent une perspective plus large. Les déterminants les plus souvent évoqués par ces modèles sont les facteurs psychosociaux, c'est-à-dire les traits de personnalité, les ressources personnelles et sociales. II demeure toutefois qu'aucun modèle n'intègre encore toutes les dimensions et tous les déterminants potentiels du «bien vieillir». <

Depuis quelques années, de plus en plus de personnes parviennent à un âge avancé, sans connaître tous les déclins jadis associés au vieillissement: on parle alors de vieillir en santé, de vieillissement réussi, de «bien vieillir». Ce credo, vieux de plus de 50 ans [1, 2], a été ramené au frontispice de l'actualité de la santé lors de la réunion annuelle de la Gerontological Society of America en 1986 [3] et, depuis, les études sur le sujet s'accumulent [4]. II suscite beaucoup d'intérêt, notamment parce qu'il conduit à une perception positive du vieillissement et offre l'alternative d'une prévention des problèmes associés à la vieillesse. Seulement, le consensus est encore loin d'être atteint sur le contenu de ce concept, ainsi que sur les facteurs éventuels qui pourraient promouvoir le bien vieillir. L'objectif de la présente synthèse est de faire le point sur la définition du bien vieillir et d'en présenter les principaux modèles conceptuels.

Article reçu le 15 juin 2005, accepté le 14 décembre 2005.

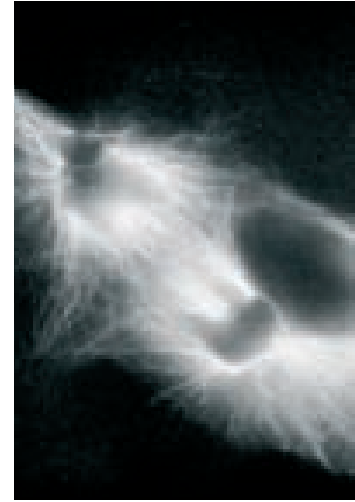

Centre de recherche,

Institut universitaire de gériatrie de Montréal, 4565, chemin Queen-Mary, Montréal (Québec), H3W IW5 Canada. marcellin.gangbe@umontreal.ca, francine.ducharme@umontreal.
Le « bien vieillir 》: essai de définition

Le bien vieillir est un concept difficile à circonscrire dans un seul énoncé, même si la plupart des auteurs mettent l'accent sur le maintien de l'autonomie fonctionnelle, surtout le fonctionnement physique, mental et social [5]. De plus, on relève au moins trois oppositions dans toute tentative de définir le bien vieillir: culturelle (0ccident/autres), de perspective (chercheurs/personnes âgées), de contenu (biomédical/holistique).

\section{Dimension culturelle du «bien vieillir 》}

Le bien vieillir est intimement lié à l'image sociale que l'on a de la personne âgée, à la place que la société lui accorde, au type de société. Ainsi, dans les sociétés occidentales fondées sur l'individualisme, où l'indépendance et l'autosuffisance de l'individu sont prônées du début de la vie jusqu'à la vieillesse, bien vieillir exige le maintien de son autonomie fonctionnelle et cognitive [6]. En revanche, dans des sociétés plus relationnelles, où la personne âgée fait partie intégrante du groupe, bien vieillir, c'est avant tout pouvoir tenir les rôles sociaux associés à son âge, indépendamment de son état de santé. Ainsi, bien vieillir en Afrique subsaharienne, c'est être dépositaire des valeurs et coutumes ancestrales [7]; au Japon, c'est être conseiller en chef pour les problèmes familiaux [8]; en Inde, c'est aller vers plus de spiritualité et de religiosité [9]; en Chine, c'est pouvoir rester en contact avec ses proches [8], etc. 
Le bien vieillir selon la perspective des acteurs

Selon la perspective des chercheurs, pour la plupart occidentaux, le bien vieillir se réfère d'abord à une liste de critères plus ou moins objectifs auxquels l'individu répond. Cela permet de distinguer trois types de vieillissement : pathologique, normal ou usuel, et réussi [10]. Et les critères du bien vieillir ou du vieillissement réussi sont alors biologiques, cognitifs ou psychosociaux.

À cette approche prescriptive des chercheurs, qui définit la manière dont les individus doivent vieillir, on peut opposer une approche émergente, qui repose sur la perception que les personnes âgées ont de leur propre vie [11]. Ainsi, pour ces dernières, bien vieillir est un processus continu de construction de sens [12], c'est l'histoire d'une vie. Cette conception de la vie comme une histoire en fait non pas une réalité linéaire, mais un processus sans fin de renégociation, au cours duquel le «moi» de l'individu et ses objectifs de vie ne cessent d'évoluer [13]. Cette renégociation continue ne se limite pas aux «moi» successifs de l'individu, mais se construit aussi avec les autres [14]. Plus qu'une simple recherche égoïste de l'intégration de soi, bien vieillir apparaît alors comme un effort continu de la co-construction d'une pluralité de «moi » [12].

\section{Approche biomédicale et holistique du bien vieillir}

Dans l'approche biomédicale, le bien vieillir est synonyme de maintien de ses capacités physiques et cognitives, ce qui explique en partie la floraison des nouvelles technologies biomédicales et génomiques et la panoplie de programmes d'intervention, tous orientés vers l'individu âgé, et qui sont destinés à le garder en forme, à renforcer sa vitalité cognitive, etc. En revanche, lorsque l'on se place dans la perspective de la santé holistique de l'Organisation mondiale de la santé, le bien vieillir recouvre des aspects aussi divers que la santé à l'âge avancé, la sécurité financière à l'âge de la retraite, la violence faite aux aînés et la qualité de leur logement. Au-delà de l'individu, c'est donc tout le contexte dans lequel il grandit et vieillit que l'approche holistique intègre.

\section{Modèles du bien vieillir}

Plusieurs modèles sont proposés pour identifier les déterminants du vieillissement : les uns, unidimensionnels, n'appréhendent le vieillissement que du point de vue d'une seule discipline scientifique, les autres, multicritères, intègrent plusieurs perspectives scientifiques.

\section{Modèles unidimensionnels}

\section{Modèles du bien vieillir biologique}

Ces modèles sont fondés sur la compression de la morbidité et la longévité. La conception de la compression de la morbidité part de la prémisse que certaines maladies dégénératives sont inhérentes au vieillissement normal. Alors, bien vieillir, ce n'est pas seulement accroître le nombre des personnes qui vivent à un âge avancé, mais c'est aussi retarder le plus possible l'apparition des maladies associées à l'âge [15].

La conception fondée sur la longévité part, quant à elle, du fait que l'âge maximum auquel l'on peut vivre est stable et dépend de l'espèce considérée. Vivre jusqu'à l'âge de 100 ans et au-delà est alors considéré comme bien vieillir [16]. Ces modèles du bien vieillir fondés sur la longévité ont donné lieu à des études du phénotype des centenaires et ont identifié certains fac- teurs qui seraient associés à la longévité [17]. Les facteurs souvent évoqués sont la distribution de la masse adipeuse et le métabolisme, les fonctions immunes et les fonctions cognitives [18-20]. Des études de laboratoire menées sur la drosophile, la souris et la levure ont permis d'identifier une vingtaine de gènes associés à 4 déterminants de la longévité, soit le contrôle métabolique, la résistance au stress, la dérégulation génétique et la stabilité génétique [17].

\section{Modèles du bien vieillir cognitif}

Ces modèles sont fondés sur les différences de performance cognitive entre les individus. En effet, selon l'approche dite normative, la performance d'une personne âgée est comparée à des données normatives obtenues à partir d'un groupe de référence du même âge : les scores de celle qui vieillit bien ou qui a bien vieilli se situent au-dessus des valeurs normatives [16]. Dans l'approche fondée sur l'âge, le bien vieillir d'un individu se mesure à une performance supérieure (de 2 ou 3 écarts types) à la moyenne du groupe de sujets auquel il appartient. Enfin, dans l'approche fondée sur les différences d'âge, les personnes âgées sont comparées à un groupe de personnes jeunes : vieillit bien la personne âgée qui a une performance comparable à celle des jeunes; elle aura maintenu ses capacités cognitives à travers les âges [16]. Les déterminants d'un déclin cognitif significatif avec l'âge sont une exposition prolongée aux hormones du stress, une dérégulation hormonale due à la ménopause/andropause, des conditions de santé défavorables comme l'hypertension artérielle et le diabète, ou encore des modes de vie à risque tels que l'éthylisme [21-25].

\section{Modèles du bien vieillir psychosocial}

Ces modèles mettent l'accent sur les interactions sociales, la satisfaction face à la vie et le bien-être comme principales sources du bien vieillir. Plusieurs courants de pensée leur sont associés. Pour la théorie de l'activité, bien vieillir suppose que l'individu demeure actif et bien intégré à la société, compte tenu de ses rôles passés et présents, mais aussi en fonction des normes sociales qui le classent comme un élément utile à la société. Pour la théorie du désengagement mutuel de l'individu et de la société, la personne qui vieillit bien se contente d'être, cesse d'agir et devient plus intensément auto-intégrée, voire égocentrique. Selon la théorie socio-environnementale, bien vieillir suppose que l'individu dispose de ressources d'activité suffisantes, en termes de santé, de solvabilité financière et de soutien social pour être capable de répondre aux attentes de son nouveau contexte social. Alors que les théories d'activité et de désengagement social demandent toutes deux de vieillir en phase avec la société, la théorie de la continuité affirme que bien vieillir est d'abord une évolution personnelle, qui se manifeste par l'intégration continue de soi et s'opère par la constance du sens donné à soi, plutôt que par une substitution d'activités (théorie de l'activité), le retrait (théorie du désengagement) ou par une modification de contextes (théorie socio-environnementale) [12]. Ces modèles ont souvent mis l'accent sur les traits de per- 
sonnalité et les ressources personnelles ou sociales comme déterminants majeurs du bien vieillir.

Les traits de personnalité reflètent ce que nous avons de plus constant et de plus stable en termes de degré d'affectivité et de comportements. Plusieurs des modèles qui en résultent et en ont été développés, retiennent deux à cinq dimensions [26] : les traits les plus fréquemment associés à la santé physique et mentale sont le névrosisme, l'extraversion, l'ouverture à l'expérience, la tendance à être agréable et le fait d'être consciencieux [27, 28].

Les ressources personnelles ne sont pas innées, mais peuvent être apprises et mobilisées [29]. Ainsi, le sentiment d'auto-efficacité ou la croyance d'une personne en ses capacités à mener à bien un aspect particulier de sa vie est liée à l'estime de soi et à différents indicateurs du bien vieillir [30]. Un faible sentiment de contrôle sur les événements de la vie détermine plus de problèmes de santé chroniques, plus de perte d'autonomie et une mauvaise santé autodéclarée [31]. Le rôle de la résilience, c'est-à-dire de la capacité de la personne à «rebondir» face aux épreuves de la vie, a été souligné dans le rétablissement des personnes après une maladie ou une perte grave [32]. Les stratégies d'adaptation (coping) sont les efforts cognitifs et comportementaux déployés pour faire face aux épreuves de la vie (pertes, deuils, etc.) et aux stresseurs chroniques de l'existence, telle la maladie chronique. Les stratégies d'adaptation sont du type résolution de problème (recherche d'information, recherche de soutien social lorsque la situation peut être modifiée) ou gestion des émotions (lorsqu'une situation n'est pas modifiable), et sont associées au bien-être et à la qualité de vie [33].

Les ressources sociales ou environnementales sont les ressources du milieu sur lesquelles les individus et groupes sociaux peuvent compter pour faire face aux circonstances difficiles de la vie. Par exemple, plusieurs études ont montré qu'un réseau de relations étendu, des interactions sociales positives, la participation à plusieurs activités sociales, le fait d'être marié, et une vie en communauté, comme c'est surtout le cas dans les kibbutz israéliens, non seulement protègent contre la dépression et les limitations fonctionnelles, mais sont aussi associés à une plus grande vitalité, une meilleure perception de la santé, un nombre réduit de symptômes dépressifs et de problèmes de santé chroniques et une plus faible probabilité de décéder [34-35].

\section{Modèles multicritères}

\section{Modèle tridimensionnel de Rowe et Khan}

Selon Rowe et Khan [4], le bien vieillir est un état, une condition objective et mesurable à un moment donné, un état meilleur que celui du vieillissement normal. Le bien vieillir inclut alors trois composantes principales et interreliées: l'absence de maladies ou de facteurs de risque, un niveau de fonctionnement physique et cognitif élevé et une vie active sur les plans occupationnel et social. Les études menées dans le contexte de ce modèle asso- cient le bien vieillir à une variété de facteurs : biologiques, sociodémographiques, psychologiques, comportementaux et relationnels [36-38].

\section{Modèle de sélection-optimisation-compensation de Baltes et Baltes}

Baltes et Baltes [39] soutiennent que les individus cherchent constamment à exercer un contrôle sur leur vie en usant d'une stratégie à trois composantes : la sélection, l'optimisation et la compensation. La sélection a trait à la définition et au choix d'objectifs, l'optimisation est le choix et l'application des moyens les meilleurs pour atteindre ces objectifs. La compensation se réfère, quant à elle, à l'adoption de moyens de substitution lorsque les moyens initiaux ne sont plus disponibles, ou se révèlent inefficaces. Dans cette approche théorique, il est reconnu que les compétences de l'individu se réduisent avec l'âge, et que bien vieillir, c'est tirer le meilleur parti de ce qui reste ou qui pourrait être disponible en utilisant des stratégies de sélection-optimisationcompensation. Très proche du modèle psychosocial, il met davantage l'accent sur les attributs individuels tels que la motivation, la capacité d'adaptation et la résilience comme déterminants majeurs du bien vieillir.

\section{Modèle du fossé structurel de Riley et Riley}

Riley et Riley [40] se sont peu appesantis sur le sens du bien vieillir. Leur modèle est un complément des deux modèles précédents, qui mettent davantage l'accent sur l'individu comme principal agent de son bien vieillir. En effet, ces auteurs se sont intéressés aux contraintes sociétales qui pourraient entraver le bien vieillir des individus. Pour eux, la structure de la population a profondément changé, sans que les structures sociales n'évoluent pour s'adapter à cette nouvelle réalité. De plus en plus d'hommes et de femmes vivent plusieurs années sans incapacités après l'âge nominal de la retraite, mais les normes et structures sociales qui régissent le travail, la vie de famille, ou les loisirs sont demeurées celles du XIX ${ }^{e}$ siècle et confinent souvent ces personnes dans l'oisiveté. Cet écart entre les exigences et opportunités de l'environnement et les capacités de l'individu provoque un fossé structurel (the structural lag) et compromet le bien-être des personnes âgées. Pour ces auteurs, l'environnement de l'individu joue un rôle déterminant dans son bien vieillir.

\section{Conclusions}

Du bien vieillir, nous avons présenté les principaux modèles théoriques. Les modèles unidimensionnels ont la faiblesse fondamentale de n'envisager le bien vieillir que dans une seule perspective. Les modèles multicritères présentent aussi plusieurs lacunes. On peut ainsi reprocher au modèle de Rowe et de Khan sa conception un peu trop élitiste du bien vieillir, et le poids excessif qu'il met sur les facteurs individuels. Le modèle de Baltes et Baltes, quant à lui, met un accent exclusif sur les compétences individuelles, sur les traits de personnalité difficiles à mobiliser et à acquérir chez ceux qui en sont dépourvus. Le modèle de Riley et Riley, enfin, passe sous silence les facteurs psychosociaux et même l'essentiel des déterminants sociaux et économiques généralement associés à l'état de santé, pour n'évoquer que la stagnation de certaines structures sociales comme frein au bien vieillir. L'idéal serait d'intégrer dans un même cadre conceptuel ces différents modèles. En effet, même si la présente recension des écrits s'est surtout attardée à montrer le rôle des facteurs psychosociaux dans la promotion du bien vieillir, on ne saurait minimiser les effets nécessairement positifs de structures de facilitation. Beaucoup reste donc à faire avant que les actions de promotion du bien vieillir n'intègrent les déterminants biologiques, psychosociaux et structurels. $\diamond$ 


\section{SUMMARY}

\section{Concepts and models of well aging}

For a few years, the image associated with the ageing process has been more positive : expressions such as «successful aging », «well aging » or «healthy aging » are more frequently used in relation to aging. However, there is still a lack of consensus on this appealing and challenging concept. Therefore, we present an overview of its definition, psychosocial determinants and conceptual models. We report that the meaning of the concept varies according to the cultural context (individualistic/relational societies), to the actors' perspectives (researcher/elderly) and according to the dominant approach (biomedical/holistic). Several models have also been identified : some are specific to a scientific domain and rely on a unique marker of well aging; others are multicriterion and embrace a broader field. Psychosocial factors are the most frequent determinants addressed by models. Among these factors, social and personal resources can be mobilized and learned, contrarily to the less modifiable personality traits. In summary, the «well aging » framework offers a unique opportunity to identify and to reinforce positive aspects in the aging process. However, the integration of the various models, more complementary than opposite, into only one meta-model remains a task to be done by researchers for a better effectiveness of «well aging » promotion programs. $\diamond$

\section{REMERCIEMENTS}

Cet article a été réalisé en partie grâce au soutien financier du groupe de recherche interdisciplinaire SOLIDAGE par l'intermédiaire d'une bourse d'études post-doctorales accordée au premier auteur.

\section{RÉFÉRENCES}

1. Havighurst RJ. Successful aging. The Gerontologist $1961 ; 1: 8-13$.

2. Hendricks J, Achenbaum A. Historical development of theories of aging. In : Bengtson VL, Schaie KW, eds. Handbook of theories of aging. New York: Springer Publishing, 1999: 21-39.

3. Fisher BJ. Successful aging, life satisfaction and generativity in later life. Int J Aging Hum Dev $1995 ; 41: 239-50$.

4. Rowe JW, Khan RL. Successful aging. New York: Random House (Pantheon), 1998 : 378 p.

5. Von Faber M, Bootsma-van der Wiel A, van Exel $\varepsilon$, et al. Successful aging in the oldest old. Arch Intern Med $2001 ; 161: 2694-700$.

6. Markus HR, Kitayama S. Culture and self : implications for cognition, emotion, and motivation. Psychol Rev $1991 ; 98: 224-53$.

7. Diop AM. The place of the elderly in African society. Impact Science Soc $1989 ; 153: 93-8$.

8. Frazier CL, Glascock AP. Aging and old age in cross-cultural perspective. In : Adler LL, Gielen UP, eds. Cross-cultural topics in psychology. Westport, CT : Praeger, 1994 : 103-11.

9. Holmes $\varepsilon R$, Holmes LD. Other cultures, elder years. Thousand Oaks, CA : Sage Publications, $1995: 336 \mathrm{p}$.

10. Rowe JW, Khan RL. Human aging : usual and successful. Science $1987 ; 237: 143-9$.

11. Keating N. Introduction : perspectives on healthy aging. Can J Aging $2005 ; 24: 3-7$.

12. Chapman SA. Theorizing about aging well : constructing a narrative. Can J Aging $2005 ; 24: 8-18$.

13. Coleman PG, Ivani-Chalian C, Robinson M. Self and identity in advanced old age : validation of theory through longitudinal case analysis. J Pers $1999 ; 67: 819-49$.

14. Bruner J. Self-making narratives. In : Fivush R, Haden $C A$, eds. Autobiographical memory and the construction of a narrative self: development and cultural perspectives. Mahwah, NJ : Lawrence Erlbaum Associates, 2003 : 209-25.

15. Fries JF. Aging, natural death, and the compression of morbidity. N Engl J Med $1980 ; 303: 130-5$.

16. Lupien SJ, Wan N. Successful ageing : from cell to self. Philos Trans R Soc Lond B Biol Sci 2004; $359: 1413-26$

17. Perls T, Kunkel LM, Puca AA. The genetics of exceptional human longevity. J Am Geriatr Soc 2002 ; $50: 359-68$.

18. Paolisso G, Gambardella A, Balbi V, et al. Body composition, body fat distribution, and resting metabolic rate in healthy centenarians. Am J Clin Nutr $1995 ; 62: 746-50$.

19. Franceschi C, Monti D, Sansoni P, Cossarizza A. The immunology of exceptional individuals : the lesson of centenarians. Immunol Today $1995 ; 16: 12-6$.
20. Hagberg B, Bauer Alfredson B, et al. Cognitive functioning in centenarians : a coordinated analysis of results from three countries. J Gerontol B Psychol Sci Soc Sci $2001 ; 56$ : P141-51.

21. Lupien SJ, Fiocco A, Wan $\mathrm{N}$, et al. Stress hormones and human memory function across the lifespan. Psychoneuroendocrinology $2005 ; 30: 225-42$.

22. Atwood CS, Meethal SV, Liu T, et al. Dysregulation of the hypothalamicpituitary-gonadal axis with menopause and andropause promotes neurodegenerative senescence. J Neuropathol Exp Neurol 2005; 64 : 93-103.

23. Papademetriou V. Hypertension and cognitive function. Blood pressure regulation and cognitive function: a review of the literature. Geriatrics $2005 ; 60: 20-4$.

24. Launer LJ. Diabetes and brain aging : epidemiologic evidence. Curr Diab Rep $2005 ; 5: 59-63$.

25. Harper C, Matsumoto I. Ethanol and brain damage. Curr Opin Pharmacol 2005 ; $5: 73-8$.

26. John $O P$. The «big five » factor taxonomy : dimensions of personality in the natural language and questionnaires. In : Pervin LA, ed. Handbook of personality: theory and research. New York: Guilford, 1990 : 66-100.

27. Hooker K, Monahan DJ, Bowman SR, et al. Personality counts for a lot: predictors of mental and physical health of spouse caregivers in two disease groups. J Gerontol B Psychol Sci Soc Sci 1998; 53 : P73-85.

28. Amelang $M$. Using personality variables to predict cancer and heart disease. EurJ Personality 1997 ; $11: 319-42$

29. Ducharme F. Transitions de vie et vieillissement... des facteurs protecteurs? Vie et Vieillissement $2003 ; 2: 15-7$.

30. Eason L. Concepts in health promotion : perceived self-efficacy and barriers in older adults. J Gerontol Nursing 2003 ; 29 : 11-9.

31. Chipperfield JG, Campbell DW, Perry RP. Stability in perceived control : implications for health among very old community-dwelling adults. J Aging Health $2004 ; 16$ : 116-47.

32. Felten BS, Hall JM. Conceptualizing resilience in women older than 85 overcoming adversity from illness or loss. J Gerontol Nurs 2001 ; 27 : 46-53.

33. Lazarus R, Folkman S. Stress, appraisal and coping. New York: Springer, $1984: 446 \mathrm{p}$.

34. Cornman JC, Goldman N, Glei DA, et al. Social ties and perceived support : two dimensions of social relationships and health among the elderly in Taiwan. J Aging Health $2003 ; 15: 616-44$

35. Blumstein T, Benyamini Y, Fuchs $Z$, et al. The effect of a communal lifestyle on depressive symptoms in late life. J Aging Health 2004 ; 16 : 151-74

36. Seeman $T \varepsilon, M c \varepsilon w e n B S$, Singer $B H$, et al. Increase in urinary cortisol excretion and memory declines: MacArthur studies of successful aging. J Clin Endocrinol Metab $1997 ; 82: 2458-65$.

37. Kubzansky LD, Berkman LF, Seeman TE. Social conditions and distress in elderly persons : findings from the MacArthur studies of successful aging. J Gerontol $B$ Psychol Sci Soc Sci $2000 ; 55$ : P238-46.

38. Seeman TE, Lusignolo TM, Albert M, Berkman L. Social relationships, social support, and patterns of cognitive aging in healthy, high-functioning older adults: MacArthur studies of successful aging. Health Psychol 2001 ; $20: 243-55$.

39. Baltes PB, Baltes MM. Psychological perspectives on successful aging: The model of selective optimization with compensation. In : Baltes PB, Baltes MM, eds. Successful aging : perspectives from the behavioural sciences. Cambridge : Cambridge University Press, 1990 : 1-34.

40. Riley MW, Riley JW. Structural lag: past and future. In : Riley MW, Khan RL, Foner A eds. Age and structural lag. New York: Wiley, $1990: 15-36$.

\section{TIRÉS À PART}

F. Ducharme 